\title{
Bayesian Estimation of Hazard Models of Airline Passengers' Cancellation Behavior
}

Esther Chiew, Cornell University, School of Civil and Environmental Engineering, 301 Hollister Hall, Ithaca, NY 14853 Email: wc437@ cornell.edu

Ricardo A. Daziano, (corresponding author), Cornell University, School of Civil and Environmental Engineering, 301 Hollister Hall, Ithaca, NY 14853 Email: daziano@ cornell.edu

Laurie A. Garrow, Georgia Institute of Technology, School of Civil and Environmental Engineering, 790 Atlantic Drive, Atlanta, GA 30332-0355, Ph: (404) 385-6634, Email: laurie.garrow@ce.gatech.edu

For submittal to Transportation Research Part A

Last update: Feb 18, 2016 


\begin{abstract}
This study explores the use of Bayesian methods to estimate hazard models of airline passengers' cancellation behavior. We show how the discrete time proportional odds (DTPO) cancellation model can be rewritten as an equivalent fixed parameter discrete choice model that can be easily estimated using Bayesian methods and extended to random parameters that account for unobserved heterogeneity. The use of Bayesian methods allows us to address several limitations of existing airline cancellation models. First, because of the random parameter reformulation, it is possible to calculate individual-specific cancellation probabilities. Second, unlike existing DTPO models that forecast average cancellation probabilities only, our model can be used to forecast both means and a measure of variance (credible intervals) associated with an individual's cancellation probability. We apply the Bayesian estimation method to a dataset of tickets purchased over a two-year period by employees of a university in Atlanta, Georgia. During this time period, the major carrier in Atlanta terminated an agreement in which it allowed employees to purchase discounted fares that could be refunded or exchanged without a fee. The data allow us to investigate how passenger cancellation behavior changed when these fares were discontinued. Cancellations are reduced on average $3.3 \%$ when customers must pay to exchange their tickets. For a simulated hypothetical flight the coefficient of variation of cancellation is $43 \%$ when the state rate was offered, and $83 \%$ without state rates.
\end{abstract}

Keywords: air travel demand; cancellation probabilities; Bayes estimation; credible intervals 


\section{Introduction and Motivation}

Airlines use revenue management models to determine the number of seats associated with a set of prices to make available to customers (Brumelle and McGill, 1994; Oliveira, 2003; Boyd, 2004; Boyd et al., 2004; Lieberman, 2004; Dunleavy and Westermann, 2005). Since some customers cancel their tickets prior to departure or fail to show up on the day of departure, airlines overbook - or sell more seats than the capacity of the flight - to reduce the number of empty seats on flights when there is demand for those seats (Ratliff, 1998). Cancellation and noshow models are used to determine overbooking levels (Chatterjee, 2001). The difference between cancellation and no-show models relates to when the airline knows passengers do not intend to travel. Cancellation models are used to forecast how many passengers will cancel or change their flight plans prior to departure. No-show models are used to forecast the number of passengers who have tickets on the day of departure, but fail to show for their flights.

Cancellation and no-show models are critical components of demand forecasting systems. Operating passenger revenues in 2014 for Delta Air Lines was \$35.0 billion (Delta Air Lines, 2015a), whereas that of JetBlue Airways was \$5.3 billion (JetBlue, 2015). Given this large revenue base, even small improvements in forecasting accuracy can translate to millions of dollars in incremental annual revenues for an airline. Talluri and van Ryzin (2004a) estimate that a 20 per cent reduction in demand forecast error translates to a 1 per cent increase in revenues. Lee (1990) estimated these benefits to be higher, i.e., that for the airline industry, a 10 per cent improvement in forecasting accuracy contributes up to a 3 per cent increase in revenues. Improving cancellation and no-show forecasts also benefits consumers. These benefits occur through reductions in the number of customers denied boarding and better alignment of supply and demand. 
Given the critical role that cancellation and no-shows play in determining overbooking levels, there has been continual interest in both: (1) exploring ways to reduce no-show and cancellation rates; and, (2) developing better forecasting models. As noted by Belobaba, lower no-show rates "inherently mean that the no-show numbers are less variable, so it makes these statistical overbooking models perform better" (De Lollis and Hansen, 2005). The increased use of online channels (and, specifically the requirement that an individual provide a form of payment at the time of booking) helps lower no-show rates (Garrow, 2004; De Lollis and Hansen, 2005). Industrywide, Belobaba reports that the no-show rate was approximately 20 per cent in the late 1990s and 12 per cent in the mid-2000s (De Lollis and Hansen, 2005).

Cancellations can be reduced by implementing business policies that restrict the ability of passengers (or make it prohibitively costly for them) to make changes to their ticket. Domestic U.S. carriers have increased exchange fees multiple times over the past decade. For example, Delta Air Lines imposed an exchange fee of \$200 for domestic tickets as of January, 2015 (Delta Air Lines, 2015b), an increase of $\$ 50$ from the fee it charged as of May, 2010 (Garrow et al., 2012). As a result, ticketing fees expressed as a percent of total operating cost increased from 2000 to 2009 for both representative low cost carriers (Frontier and JetBlue increased from 1.3 to 3.2 per cent) and major network carriers (Alaska, United, and Northwest increased from 1.0 to 2.5 per cent) (see Garrow et al., 2012, Table 4). In addition, some airlines offer highly discounted tickets that cannot be refunded or exchanged. Alaska Airlines has done this since at least 2010 (Garrow et al., 2012) and Delta Air Lines recently announced the creation of a "basic economy" product that cannot be refunded or exchanged (Delta Air Lines, 2014).

The business policies above apply to tickets sold to the general public. Additional policies specific to Corporate Volume Agreements (CVAs) can also help lower cancellation

\footnotetext{
${ }^{1}$ The same logic applies for cancellation rates.
} 
rates. A CVA is an agreement between an airline and a public or private institution (referred to as a "corporation") in which the corporation agrees to purchase a certain volume of tickets with the airline in exchange for a discount and/or other benefits, such as the ability to exchange or refund tickets without a fee. Airlines that waive exchange fees as part of CVAs may see an increase in the cancellation rates and/or an increase in the variability of their cancellation rates.

In addition to implementing business policies that can help lower no-show and/or cancellation rates, the aviation and research communities have explored ways to improve their forecasting models. With respect to cancellation models, it is common to model that number of bookings surviving until departure using a binomial distribution (Talluri and van Ryzin, 2004a). This distribution is convenient, due to the ability to calculate both a mean and variance associated with a cancellation forecast. Alternative approaches based on a hazard model have been proposed (Iliescu et al., 2008; Graham et al., 2010). The use of a hazard model for forecasting cancellation rates is conceptually appealing because it can capture the fact that the intensity of the cancellation process is influenced both by the time of ticket purchase and the time before flight departure. Specifically, higher cancellation rates are observed for recently purchased tickets and for tickets whose associated flight departure dates are near. However, one of the key limitations of the hazard model proposed by Iliescu and colleagues (2008) is that the model can be used to forecast average cancellation probabilities - but not the variance associated with the forecast. Many airline revenue management systems require as inputs information about both the mean and variance of cancellation forecasts; thus, industry adoption of the hazard model has been limited.

Our paper contributes to the literature in three key ways. First, we reformulate the hazard model proposed by Iliescu and colleagues (2008) as an equivalent discrete choice model (cf. 
Garrow, 2010; Talluri and van Ryzin, 2004b) so that its parameters can be easily estimated using Bayesian methods. Second, we extend our formulation to include random parameters. This allows us to forecast conditional individual-level cancellation probabilities and confidence/credible intervals (which can be viewed as a measure of variance) and incorporate information about repeat purchases from the same individual. To the best of our knowledge, this is the first time that an approach to calculate cancellation probabilities for individual customers has been reported in the literature.

Whereas our first two contributions relate to methodological advancements to improve cancellation forecasting, our third major contribution relates to assessing the impact of business policy changes on reducing cancellation rates. We apply the Bayesian estimation approach to a dataset that contains two years of ticket transactions (2011-2013) for individuals employed by the Georgia Institute of Technology (Georgia Tech). During this time period, Delta Air Lines terminated its CVA with Georgia Tech. This provides us with the opportunity to examine how individuals' cancellation behavior changed when "state rates" that were originally offered through the CVA were no longer available. State rates are defined as discount fares with no fees associated with refunds and exchanges.

The remainder of the paper is organized as follows. The next section reviews airline cancellation models. Sections 3 and 4 describe our methodology and data, respectively. Section 5 presents results, including how the discontinuation of the state rates affected individuals' cancellation behavior. Section 6 presents two key applications of our results. The first shows how individual-specific cancellation probabilities, and their associate credible intervals, can be calculated. The second demonstrates the calculation of the mean and variance of flight-level 
cancellation rates, which would be of more interest to airlines. The paper concludes with a discussion of limitations and directions for future research.

\section{Review of Airline Cancellation Models}

Iliescu et al. (2008) provide an overview of cancellation models. They note that there are two main categories of cancellation models reported in the literature. The first category is used to predict the probability that a ticket purchased by a passenger $D$ days from flight departure will survive until departure. The binomial model is commonly used to model the number of tickets surviving until departure (Talluri and van Ryzin, 2004a). However, the binomial distribution imposes strong assumptions, most notably that cancellation probabilities are "memoryless" and depend only on the time to flight departure and not when the ticket was originally issued.

Based on a sample of tickets from KLM, Westerhoff (1997) provides evidence that the memoryless property of cancellation probabilities is violated. To help address this limitation, Westerhoff proposed a second category of cancellation models that predict the probability a ticket will survive from one period to the next. That is, $D$ is divided into distinct time periods (usually days) and the model predicts the probability that a ticket purchased $D$ days from departure will survive from one day $(D)$ to the next $(D-1)$.

Iliescu et al. (2008) leverage Westerhoff's idea by using time-to-event models, specifically a discrete time proportional odds (DTPO) model, to predict cancellations. As a survival analysis method, this model was applied to calculate the hazard probability, i.e. the conditional probability that a ticket would be cancelled on a certain day given that it had survived until that day. The DTPO model proposed by Iliescu et al. (2008) builds on prior research in two key ways. First, whereas most survival models consider a single time dimension, their model accounts for multiple time dimensions by accommodating time-varying covariates. 
This is important, as it allows them to model the influence of both the time from ticket purchase and the time before flight departure on the intensity of the cancellation process. By including both days from ticketing and days from departure in the model, the DTPO model is able to capture the fact that higher cancellation rates are observed for recently purchased tickets and for tickets whose associated flight departure dates are near. Due to the discrete nature of the timescale used, they were able to test for different distributional structures of the baseline cancellation rate. Second, their DTPO model builds on prior research by empirically showing how cancellation rates are influenced by ticket characteristics (market, carrier, fare, outbound departure date, number of passengers traveling together, indicator as to whether the trip involves a Saturday night stay). However, although their DTPO model allows for the analysis of the effect of ticket heterogeneity on cancellation probabilities, it assumes fixed parameters, i.e., that the ticket heterogeneity is fully contained within the covariates used, and that its effect on cancellation probability is separate from that of time. This limitation can be addressed through using a random parameter formulation.

Using a distinct ticketing database, Graham et al. (2010) validated the key finding from Iliescu et al.'s work, namely that the cancellation process is influenced by both the time of issue and time until departure. The ticketing database used by Graham and colleagues allowed the authors to: (1) examine the impact of "state rates" on business travelers' cancellation behavior; and, (2) track individual passengers over time. Their study found that individuals who purchased state rates were 2.0 times more likely to cancel their tickets and that frequent travelers were 1.4 times more likely to cancel their tickets than non-frequent travelers. However, with respect to the second finding, they did not directly incorporate individual passengers' past cancellation history 
into the DTPO model, but simply divided travelers into two groups (frequent versus infrequent) and determined the percentage of tickets that were cancelled.

To the best of our knowledge, all of the airline cancellation models reported in the literature have been based on classical estimation methods for models with fixed parameters. Estimating the DTPO model via Bayesian methods provides two key opportunities: the ability to (1) easily incorporate individuals' historic cancellation behavior directly into the DTPO model; and, (2) construct the posterior density and credible interval of the individual likelihood of a passenger having performed a certain event, namely cancelling or using a ticket. In this paper we focus on the second opportunity by taking into account uncertainty in the determination of cancellation probabilities.

Interval estimates are important not simply as a statistical result, but also because the average probability of a passenger cancelling a ticket does not hold much value on its own. Since probability is random, simply knowing the mean of this probability does not describe how much this probability can be trusted or expected to be true ${ }^{2}$. Practically, knowing the interval estimates also allows the airline to know how reliably they can use the cancellation probability in their revenue management models, and also to incorporate appropriate measures of uncertainty.

Our paper contributes to the literature by using Bayesian estimation methods to construct credible intervals. Conceptually, the hierarchical Bayes model is appealing because it can generate conditional individual-specific parameters, and thus individual-specific probabilities. To date, the DTPO models used in the literature forecast average cancellation probabilities. Since the Bayesian methods take draws from the posterior distribution of the parameters, post

\footnotetext{
${ }^{2}$ As an example, consider two cancellation forecasts, where both are Normally distributed with means of 10 but the first has a variance of 0.5 and the second a variance of 3 . In the first case, the airline can more confidently set an overbooking level at 10, as the probability that the number of cancellations is less than 10 (and that more individuals show up for their flights than was predicted, leading to denied boardings) is small. In the second case, the airline would likely set the overbooking level less than 10 to help reduce the number of expected denied boardings.
} 
processing methods can be used to obtain individual-specific credible intervals for these probabilities.

\section{Methodology}

This section reviews the DTPO model and describes how we reformulated it to estimate its (fixed) coefficients using Bayesian methods. Section 3.3 presents an extension for estimating random coefficients, which allows us to determine individual-level cancellation probabilities that incorporate prior history. The methodology section concludes with a description of how credible intervals for individual-level cancellation probabilities are calculated.

\subsection{Discrete Time Proportional Odds Model}

The DTPO model partitions the time-to-event (where the event is either the ticket being cancelled or the passenger using the ticket and departing) of the $i^{\text {th }}$ ticket $\left(T_{i}\right)$ into $k$ disjoint time intervals $\left(t_{0}, t_{1}\right],\left(t_{1}, t_{2}\right], \ldots,\left(t_{k-1}, t_{k}\right]$, where $\left(t_{0}, t_{1}, \ldots, t_{k}\right)$ identify the days from issue of the ticket, and $t_{0}$ and $t_{k}$ are the issue date and the time of event, respectively. Additionally, the discrete hazard probability (that is, the probability of the cancellation event) is given by $h_{i j}=P\left(T_{i}=j \mid T_{i}\right.$ $\geq j$ ). By using conditional probability theory, the likelihood function of the entire sample can be written explicitly. In particular, suppose we have the choice indicator variable $y_{i j}$, where

$$
y_{i j}=\left\{\begin{array}{l}
1, \text { if ticket } i \text { is canceled } j \text { days from issue } \\
0, \text { otherwise. }
\end{array}\right.
$$

Then the likelihood function of the entire sample becomes

$$
\ell=\prod_{i=1}^{n} \prod_{j=1}^{k} h_{i j}^{y_{i j}}\left(1-h_{i j}\right)^{\left(1-y_{i j}\right)}
$$

The DTPO model incorporates the ticket characteristics (known as covariates) into the model through the following formulation. For a set of covariates $\boldsymbol{x}_{i}$, the hazard probability $h_{i j}$ is 


$$
\log \left(\frac{h_{i j}}{1-h_{i j}}\right)=\Psi_{i j}+\beta_{1} x_{i j 1}+\beta_{2} x_{i j 2}+\cdots+\beta_{l} x_{i j l}
$$

where $\Psi_{i j}$ is the baseline hazard function, $j=1,2, \ldots, k$ observations and $l$ is the number of covariates. Using this formulation, the DTPO model can be solved for the vector of parameters $\boldsymbol{\beta}$.

However, since the exact time at which the ticket is cancelled is known and can be written using a binary variable ( $y_{i j}$ above), the likelihood function is equivalent to that of a binary logistic regression model. Recall that the logistic regression model is described as follows:

$$
\begin{aligned}
& \boldsymbol{y}_{\boldsymbol{i}}^{*}=\boldsymbol{x}_{i}^{\prime} \boldsymbol{\beta}+\boldsymbol{\varepsilon}, \text { where } \boldsymbol{\varepsilon} \sim \operatorname{Logistic}(0,1) \\
& y_{i j}= \begin{cases}1, & \text { if } y_{i j}^{*}>0 \\
0, & \text { otherwise }\end{cases}
\end{aligned}
$$

Then the probability that $y_{i j}=1$ given covariates $\boldsymbol{x}_{\boldsymbol{i}}, \boldsymbol{p}_{\boldsymbol{i}}=P\left(\boldsymbol{y}_{\boldsymbol{i}}=1 \mid \boldsymbol{x}_{i}\right)=\frac{e^{\boldsymbol{x}_{i}^{\prime} \boldsymbol{\beta}}}{1+e^{\boldsymbol{x}_{i}^{\prime} \boldsymbol{\beta}}}$.

The likelihood function of the entire sample simply becomes:

$$
\ell(\boldsymbol{\beta} ; \boldsymbol{y} \mid \boldsymbol{x})=\prod_{i=1}^{n}\left(\frac{e^{x_{i}^{\prime} \beta}}{1+e^{x_{i}^{\prime} \beta}}\right)^{y_{i}}\left(\frac{1}{1+e^{x_{i}^{\prime} \beta}}\right)^{\left(1-y_{i}\right)}
$$

Note that equations (2) and (5) are equivalent if the baseline hazard function $\Psi_{i j}$ is added as a covariate. Hence the DTPO model is equivalent to that of a binary logistic regression model, which has known numerical solutions and thus makes the DTPO model analytically attractive. The ability to write the DTPO model as a binary logistic regression model provides a mechanism by which the DTPO model can be reformulated to facilitate estimation of its parameters by Bayesian methods.

\subsection{Reformulation of the DTPO model for Fixed Parameters}


To easily use Bayesian methods to solve the DTPO model, the model was reformulated as a discrete choice model that maintains the same assumptions as the DTPO model. That is, by reformulating the complex DTPO model to that of a simple logistic regression model, ${ }^{3}$ we were able to use standard software packages (such as the mlogit and gmnl functions in the $\mathrm{R}$ programming language) to solve for model parameters.

Our reformulation includes two latent variables. In particular, the cancellation data can be modeled as an individual making a choice, in each time period, of whether to cancel the ticket (alternative 1), or not (alternative 2 as an outside or opt-out option). This reformulation of the DTPO model into a sequence of binary choice situations provides an intuitive representation of the cancellation behavior of a passenger. Specifically, as the days pass from the purchase of a ticket, a passenger can decide whether or not to cancel the ticket. This is clearly modelled in the sequence of binary choices, where for every time period (day), the individual chooses between two alternatives - whether to cancel the ticket or not. In addition, since some of the covariates (in particular, those associated with the day before departure and the day after issue) are time dependent, this model represents a dynamic choice problem. Hence this model, unlike other survival models, is also able to represent multiple time dimensions due to its inclusion of timedependent covariates.

For each binary choice situation, the individual has a utility associated with each choice:

$$
\begin{aligned}
& U_{1}=\boldsymbol{x}_{\mathbf{1}}^{\prime} \boldsymbol{\beta}+\varepsilon_{1} \\
& U_{2}=\boldsymbol{x}_{\mathbf{2}}^{\prime} \boldsymbol{\beta}+\varepsilon_{2}, \quad \text { where } \varepsilon_{1}, \varepsilon_{2} \sim E V 1
\end{aligned}
$$

As is standard in random utility maximization models, only utility differences are uniquely identified, i.e., the difference in the utilities obtained from choosing alternative 1 or alternative 2. Hence the following model is achieved:

\footnotetext{
${ }^{3}$ The logistic regression model is equivalent to a binary logit model.
} 


$$
U=U_{1}-U_{2}=\left(\boldsymbol{x}_{\mathbf{1}}-\boldsymbol{x}_{\mathbf{2}}\right)^{\prime} \boldsymbol{\beta}+\left(\varepsilon_{1}-\varepsilon_{2}\right), \text { where } \varepsilon_{1}-\varepsilon_{2} \sim \text { Logistic }
$$

Setting all $\boldsymbol{x}_{2}$ variables to 0 makes this discrete choice model equivalent to the logistic regression model, and thus the original DTPO model. To the best of our knowledge, reformulating the DTPO logistic model as a choice model has not been done before. However, much of the literature which involves an opt-out alternative uses this method of setting the utility of the opt-out alternative to 0 . An opt-out alternative is where the individual taking a survey is given the option to choose either one of the scenarios portrayed or to choose none of the scenarios portrayed (Veldwijk et al, 2014). Hence, the individual "opts out" of choosing an available scenario or alternative. Many surveys include the opt-out alternative as a means of better simulating a real life situation. Representative examples in the airline context include Garrow, et al. (2007) and Brey and Walker (2011).

Using the discrete choice representation involves expanding the original data set to include the attributes of the second/opt-out alternative. Table 1 shows how the dataset is created. The table presents information for one customer, the customer's choice to cancel (choice=1) or not cancel (choice $=2$ ), and variables associated with ticket characteristics. The original dataset ${ }^{4}$ used for the DTPO model reported in Graham, et al. (2010) is shown in the non-shaded columns. The shaded columns show how the dataset has been expanded, to include ticketing characteristics for the no cancellation option (alternative 2).

[ Insert Table 1 about here ]

\subsection{Determining Individual-level Cancellation Probabilities}

\footnotetext{
${ }^{4}$ Our data is from the same source as that used in the Graham, et al. (2010) paper; however, our data represents ticket transactions for a different time period.
} 
One major contribution that this paper adds to the current literature is the application of the random parameter model specification to the cancellation model to determine individual-level cancellation probabilities. The fixed parameter formulation described above does not account for unobserved dynamics. In particular, each time partition is treated individually, and the fact that it is a single ticket partitioned over time is not taken into account. In addition, the fixed parameter formulation does not account for the fact that there are multiple tickets purchased by the same individual. Hence each sequence of binary choices is treated individually rather than seen as having been made by the same individual.

To correct this discrepancy, the random parameter model is used to specify the cancellation model. By using the random parameter model, we assume that each individual has his or her own taste parameter $\boldsymbol{\beta}_{\boldsymbol{i}}$. This varies between individuals, but is constant for all choices made by a single individual. In this way, the sequence of binary choices is now modelled as having been made by the same individual.

The random parameter model is formulated as a DTPO mixed multinomial logit. Consider that we have individuals $i=1, \ldots, n$. Each individual purchases at least one ticket, hence the total number of tickets purchased is more than or equal to $n$. As before, each individual faces two choices, each with its own associated utility as follows:

$$
\begin{aligned}
& U_{1 i t}=\boldsymbol{x}_{\mathbf{1 i t}}^{\prime} \boldsymbol{\beta}_{\boldsymbol{i}}+\varepsilon_{1 i t} \\
& U_{2 i t}=\boldsymbol{x}_{2 i t}^{\prime} \boldsymbol{\beta}_{\boldsymbol{i}}+\varepsilon_{2 i t}, \text { where } \varepsilon_{1 i t}, \varepsilon_{2 i t} \sim E V 1
\end{aligned}
$$

The subscript $t$ is also added here to account for the time dimension of the problem, as each ticket is partitioned into separate days from the date of issue to the date of an event occurring. 
The coefficients $\boldsymbol{\beta}_{\boldsymbol{i}}$ vary over individuals in the population with density $f(\boldsymbol{\beta} \mid \boldsymbol{\theta})$, where $\boldsymbol{\theta}$ represents hyperparameters. As before, we take the differences in utilities to derive the following model:

$$
U_{i t}=U_{1 i t}-U_{2 i t}=\left(\boldsymbol{x}_{1 i t}-\boldsymbol{x}_{2 i t}\right)^{\prime} \boldsymbol{\beta}_{\boldsymbol{i}}+\left(\varepsilon_{1 i t}-\varepsilon_{2 i t}\right) \text {, where } \varepsilon_{1 i t}-\varepsilon_{2 i t} \sim \text { Logistic }
$$

Since $\boldsymbol{\beta}_{\boldsymbol{i}}$ is not observed, the unconditional choice probability is thus the integral of the standard logit choice probability over all possible variables of $\boldsymbol{\beta}_{\boldsymbol{i}}$, i.e. the probability of choosing alternative 1 is

$$
P_{1 i t}=\int\left(\frac{e^{x_{1 i t}^{\prime} \beta}}{e^{x_{1 i t}^{\prime} \boldsymbol{\beta}}+e^{x_{2 i t}^{\prime} \boldsymbol{\beta}}}\right) f(\boldsymbol{\beta}) d \boldsymbol{\beta}
$$

and similarly for the probability of choosing alternative 2 .

Using Bayesian methods to solve for the random parameter model contains the following additional benefit: past ticketing history can be used as prior information. This would allow for more precise determinations of cancellation probabilities. As the ticketing history is on an individual level, the cancellation probabilities can also be presented on an individual level. The random parameter is solved by applying a Bayesian hierarchical model. As stated, each covariate has an associated coefficient that now takes on an assumed distribution, and the parameters of these distributions are solved. The procedure for solving the Bayesian hierarchical model is described in Train (2009) and described for our application in the Appendix. Whereas random parameter models can also be estimated using classical methods, the Bayesian hierarchical model has the advantage where the individual parameter values are direct results, being taken from each line of the Monte Carlo Markov Chain (MCMC) sampler.

\subsection{Constructing Credible Intervals}


Bayesian model results include multiple MCMC draws from the posterior distribution of the parameters. Via post-processing of these MCMC draws (Edwards and Allenby, 2003), credible intervals can be easily calculated for the probability of cancellation. Specifically, the probability of cancellation is calculated using each MCMC draw (say there are $R$ draws). The highest probability density interval is then taken over these $R$ draws, resulting in the credible interval of the draw.

Credible intervals have an advantage over classical confidence intervals in that the interpretation of these is more intuitive. The classical setting sees the parameter around which the confidence interval is built as being fixed, and hence a " $95 \%$ confidence interval" means that over repeated sampling of the confidence intervals, $95 \%$ of these intervals will contain this true fixed parameter. In contrast, the Bayesian approach treats the parameter as random, and thus this random parameter has a $95 \%$ chance of falling within the " $95 \%$ credible interval."

Credible intervals also provide the variance that airline revenue management systems require as input information. As mentioned in the introduction, industry adoption of the hazard model has been limited due to its inability to estimate the variances associated with the forecasted cancellation probabilities. The width of the credible interval is approximately four times the standard error of the estimated parameter, and thus the variance can be easily extracted to be used as input for airline revenue management systems. In addition, credible intervals provide for an easy visual understanding of the variance. One can easily determine if the cancellation probability is reliable by simply looking at the width of the interval estimates.

Since each ticket contains different details, the credible interval is calculated for separate individuals. These individual cancellation probabilities can also be aggregated in order to obtain the flight-level cancellation rate and its associated variance, as demonstrated in Section 6. 


\section{Data}

The dataset used contains information of 12,958 tickets purchased by a dedicated ticket agency on behalf of Georgia Institute of Technology (Georgia Tech). The analysis database contains tickets purchased from July 1, 2011 - June 27, 2013 for outbound travel dates from July 6, 2011 - July 15, 2013. Only round-trip tickets for domestic U.S. travel in which both the outbound and inbound itineraries were served by nonstop flights on Delta or AirTran were included in the analysis. Consistent with Iliescu et al. (2008), we model cancellations (which include refund and exchange events) for the outbound portion of the itinerary. We exclude cancellations for the return itinerary as passengers who are traveling are more likely to make changes to their itinerary by calling the airline directly; these changes are not captured in our analysis database (thus cancellations for the inbound itineraries are under-reported). Of the tickets purchased, 12,095 (or $93 \%)$ of the tickets were used, whereas $863(7 \%)$ were cancelled prior to departure.

The dataset contains a total of 4,820 individuals, of whom 1,782 (37\%) individuals purchased at least two tickets on separate occasions. The majority of individuals who purchased multiple tickets bought two tickets (38\%), with the remaining individuals purchasing between 3 and 66 tickets on separate occasions.

For travel occurring prior to January 1, 2013, individuals travelling on official business on behalf of the Institute were eligible to receive "state rates." These tickets, which were purchased through Delta or AirTran, allowed passengers to exchange their tickets at no charge (i.e., no exchange fees were imposed).

The dataset contains primarily information about the ticket that was purchased. In particular, only the passenger's name and a university issued ID number identify the passenger 
using that ticket. The remaining variables all describe the ticket that was purchased, as detailed in Table 2.

[ Insert Table 2 about here ]

\section{Results}

\subsection{Binary Logit Model}

Using the data as described above, a binary logit model was first estimated. This model was run on the full dataset without taking into account individuals having bought multiple tickets. Modelling the data using a binary logit model also allows us to estimate the odds ratio (OR) for each variable.

Table 3 compares the parameter estimates from both the classical and Bayesian methods of estimating the binary logit model. As can be seen the results obtained from both classical and Bayesian methods are very similar. In addition, there are a number of variables that are significant in affecting the cancellation rate. In particular, the days from issue (DFI) variables: DFI, DFI_4_7, DFI_15_21; days from departure (DFD); indicator for whether the itinerary originated in Atlanta (ATLORG); whether the ticket was a state discounted ticket (STATE); an indicator for departures that occurred in September (SEPT); and an indicator that the itinerary arrived between 9:00 PM - 9:59 PM (A21) were statistically significant with at least 95\% confidence (using a frequentist approach to hypothesis testing). There are also a number of other variables which are statistically significant at the $90 \%$ confidence level.

It is easier to understand the impact of the variables on the cancellation probability by looking at the odds ratio for each variable, which is also reported in Table 3. Cancellations are about 1.6 times more likely to occur 4-7 days from the issue date, and about 1.4 times more 
likely to occur 14-21 days from the issue date (as opposed to 22-220 days from the issue date). Similarly, tickets are more likely to be cancelled as the date of departure approaches. Tickets purchased in September are 0.6 times as likely to be cancelled (compared to tickets purchased in December). Cancellations are 0.3 times as likely to occur for flights that arrive between 9:00 PM - 9:59 PM. Tickets for flights originating from Atlanta are 1.4 times more likely to be cancelled as compared to tickets for flights arriving in Atlanta. Finally, cancellations are about four times more likely for tickets that were purchased using a state discount.

[ Insert Table 3 about here ]

\subsection{Random Parameter Model}

By identifying passengers who have bought more than one ticket with this ticket agency, we were able to estimate a random parameter, or mixed multinomial logit model to test for preference heterogeneity among passengers. Based on the results of estimating the logit model and Graham et al. (2010), we decided to test different combinations of random parameters. The first model (RP1) assumes to be random those variables that Graham et al. (2010) found to be statistically significant. The second model (RP2) assumes to be random all variables that were found to be statistically significant at the $95 \%$ confidence level in the binary logit model estimated in Section 5.1. Finally, the third model (RP3) assumes to be random all variables that were found to be statistically significant at the $90 \%$ confidence level in the binary logit model estimated in Section 5.1. All random variables assume a normal distribution, and all three random parameter models were estimated using the Bayesian method with only ticket data from passengers who purchased multiple tickets. 
The assumption that the variables are randomly normally distributed is proven accurate if the estimated standard deviations are different from zero. The $99 \%$ credible intervals of the standard deviations do not contain zero, hence affirming that random taste variation exists amongst passengers for these variables.

[ Insert Table 4 about here]

\subsection{Discontinuation of State Rates}

Table 5 details how cancellation behavior changes when state rates are no longer offered to customers. On January 1, 2013, Delta Air Lines terminated its CVA with Georgia Tech. As a result, the dataset contains tickets purchased from a time period when state rates were available (July 1, 2011 - December 31, 2012) and when state rates were not offered (January 1, 2013 July 15,2013$)$. The percentage of cancellations dropped from $7.6 \%$ to $4.3 \%$, reflecting a decrease of $3.3 \%$ when state rates were no longer offered. The results show that cancellations are, in fact, reduced by implementing policies that make it costlier for passengers to make changes to their ticket. The act of terminating the CVA resulted in passengers having to now pay a fee in order to change or cancel their ticket, hence resulting in a reduction in the cancellation of tickets.

[ Insert Table 5 about here]

\section{Applications}

It is possible to apply our results in multiple ways, and here we demonstrate how the individualspecific likelihoods of an event occurring can be calculated, as well as the variance of this

likelihood. In addition, we also demonstrate how the average number of tickets cancelled per 
flight, as well as the associated variance, can be calculated. These can all be calculated using the estimates of the random parameter model, and we choose the RP3 model results as it has the highest model log-likelihood value.

A hypothetical situation is first created where passengers book their tickets five days before the scheduled departure date. The flight is booked with Delta, and originates in Atlanta. It is scheduled to depart on a Tuesday in November at 5:15 PM, and arrive at its destination at 7:45 PM. Finally, the passenger is staying away from home for 2 nights. We create two hypothetical tickets by changing whether or not a state rate is received when purchasing the ticket. This will further reinforce the impact that receiving state rate has on the probability of cancellation.

\section{Section 6.1 Individual-specific Cancellation Probability}

Under the hypothetical tickets described above, we calculate the probability, and accompanying credible interval, that an individual will cancel his ticket on the day before departure. Figure 1 shows the mean of the probability of cancellation for 100 sampled individuals, comparing the situations where the individuals received or did not receive a state rate when purchasing their ticket. Figure 2 shows also the credible intervals for these 100 sampled individuals. Note that the same 100 individuals were sampled for both hypothetical situations.

[ Insert Figure 1 about here ]

As can be seen, the individual-specific cancellation probability drops when the state rate is not offered. The width of the credible interval likewise decreases. This reinforces the results found in Section 5 .

[ Insert Figure 2 about here ] 
We can also use results from the random parameter model to calculate the probabilities and credible intervals for a particular individual's decision process on a daily basis. Under the same hypothetical scenario as described above, the probability that this given individual would cancel his ticket on any given day from the time he purchased his flight to the day he flies (technically a no-show) is shown in Figure 3, along with the accompanying credible intervals. Again, the probability is much lower when the state rate is not offered.

[ Insert Figure 3 about here ]

The coefficient of variation $(\mathrm{CV})$, which is defined as the ratio of the standard deviation to the mean, shows how variable the data is with respect to the mean of the population. The CV of the individual's cancellation probability was calculated for each day in Figure 2, and ranges from 5.8 - 7.5. These high $\mathrm{CV}$ values, especially considering the low mean cancellation probabilities, indicate that there is great variance contained in this particular individual's cancellation probability, and thus the airline would need to incorporate greater uncertainty for this individual into their models.

\section{Section 6.2 Flight-level Cancellation Rates}

Suppose we have 100 individuals who have purchased tickets for the above flight. In order to calculate the mean cancellation rate for the flight, we first calculate the probability that each individual cancels their ticket. The number of cancellations for that flight is the sum of all the individual probabilities. This process is repeated many times, and the mean cancellation rate and associated variance for the flight can be calculated by taking the mean and variance over the trials. 
For the hypothetical flight described above when a state rate is offered, and assuming that 100 individuals have purchased tickets for that flight, the average number of cancellations is 1.38 , with a variance of $0.26(\mathrm{CV}=43.48 \%)$. In contrast, when a state rate is not offered, the average number of cancellations drops to 0.38 , with a variance of $0.10(\mathrm{CV}=83.21 \%)$. This reiterates the finding that imposing change or cancellation fees reduces the cancellation rates for airlines.

\section{Conclusion}

In this paper, we demonstrate how the DTPO model can be solved as a discrete binary choice model using Bayesian methods. In particular, we use both the binary logit and the random parameter models to specify the cancellation model. Our results show that it is important to account for and partition tickets according to the individual who purchased them, as the standard error values of the assumed Normal distribution of parameters were statistically significant. In addition, by using the Bayesian approach, we are able to easily build the credible intervals of the individual likelihoods of an event occurring. These credible intervals are of value as they show the uncertainty behind the estimate of the likelihood, allowing airlines to better be able to incorporate these estimates into their revenue management models. The widths of the credible intervals created in our examples also demonstrate the importance of reporting these variances, as the widths are often more than two times that of the estimated cancellation probability itself. In fact, the exercise of creating a ticket and looking at the cancellation probabilities is regularly carried out by airlines, and we demonstrate that, by using Bayesian methods, it is possible to easily do this for any given individual.

Not only are we able to easily report credible intervals for any individual's probability of cancelling their ticket on a certain day, we are also able to track a given individual's probability of cancellation throughout the time period between his purchase of the ticket and the scheduled 
date of departure. Again, the width of the intervals created, as well as the CV for each day, demonstrates the importance of providing a measure for the variance of the probability of cancellation, as the point estimate itself hides the degree of variability that was actually obtained.

The data used in this paper contained only information about business travelers; hence the numerical credible interval results and parameter estimates is not generally applicable to all travelers. However, this paper still holds significance: firstly, we demonstrate the importance of accounting for unobserved heterogeneity in cancellation models. Secondly, the methodology used in this paper is applicable to any data set containing such ticket characteristic data. As such, although the results obtained in this paper might only be applicable to airlines concerning their business travelers, it can be easily applied to any data involving both business and leisure passengers, for which identifying characteristics exist (Iliescu et al., 2008).

Future work in this area aims to further incorporate the available historical ticketing data. In particular, this current work is based on an estimator that only allows priors for the hyperparameters that describe the population. By taking advantage of the fact that certain individuals have purchased more than one ticket, we hope to use parts of this "history" as an informative prior for the parameters at the individual level to reduce the width of the Bayesian confidence intervals of the cancellation probabilities. This should further increase the accuracy of the Bayesian model estimates. In addition, we also hope to identify other individual variables and characteristics that can further improve the cancellation probabilities estimated.

\section{Acknowledgments}

We acknowledge and thank Stacey Mumbower for her help in preparing the estimation dataset. 


\section{References}

Belobaba, P. P. (1989). Application of a probabilistic decision model to airline seat inventory control. Operations Research 37(2): 183-197.

Boyd, E. A. (2004). Dramatic changes in distribution will require renewed focus on pricing and revenue management models. Journal of Revenue \& Pricing Management 3(1): 100-103.

Boyd, E. A. and R. Kallesen. (2004). The science of revenue management when passengers purchase the lowest available fare. Journal of Revenue \& Pricing Management 3(2): 171177.

Brey, R. and J.L. Walker (2011) Latent temporal preferences: An application to airline travel. Transportation Research Part A, 45(9): 880-895.

Brumelle, S. L. and J. I. McGill. (1993). Airline seat allocation with multiple nested fare classes. Operations Research 41(1): 127-137.

Chatterjee, H. (2001). Forecasting for cancellations. Presented at AGIFORS Reservations and Yield Management Study Group, Bangkok, Thailand.

Delta Air Lines (2014). Delta redefines cabin, upgrades premium economy options. Available online at news.delta.com/2014-12-08-Delta-Redefines-Cabin-Upgrades-PremiumEconomy-Options. Accessed January 2, 2015.

Delta Air Lines (2015a). Delta Air Lines announces December quarter results. Available online at http://news.delta.com/2015-01-20-Delta-Air-Lines-Announces-December-QuarterResults. Accessed June 16, 2015.

Delta Air Lines (2015b). Exchange fees. Available online at www.delta.com/content/www/en_US/traveling-with-us/ticket-changes-refunds/ticketchanges.html. Accessed January 2, 2015.

Dunleavy, H. and D. Westermann. (2005). Future of airline revenue management. Journal of Revenue \& Pricing Management 3(4): 380-383.

Edwards, Y. D. and G. M. Allenby (2003). Multivariate analysis of multiple response data. Journal of Marketing Research, 40(3): 321-334.

Garrow, L.A. (2004). Comparison of Choice Models Representing Correlation and Random Taste Variation: An Application to Airline Passengers' Rescheduling Behavior. PhD dissertation, Northwestern University.

Garrow, L. A. (2010). Discrete Choice Modelling and Air Travel Demand: Theory and Applications. Ashgate Publishing Limited, England. 
Garrow, L.A., S. Hotle, and S. Mumbower (2012). Assessment of product debundling trends in the U.S. airline industry: Customer service and public policy implications. Transportation Research Part A 46(2): 255-268.

Garrow, L. A., S. P. Jones and R. A. Parker. (2007). How much airline customers are willing to pay: An analysis of price sensitivity in online distribution channels. Journal of Revenue \& Pricing Management 5(4): 271-290.

Graham, R. J., L. A. Garrow and J. D. Leonard. (2010). Business travelers' ticketing, refund, and exchange behavior. Journal of Air Transport Management 16(4): 196-201.

Iliescu, D. C., L. A. Garrow and R. A. Parker. (2008). A hazard model of US airline passengers' refund and exchange behavior. Transportation Research Part B 42(3): 229-242.

JetBlue (2015). JetBlue Announces Fourth Quarter and Full Year Results. Available online at http://otp.investis.com/clients/us/jetblue_airways/usn/usnewsstory.aspx?cid=981\&newsid=28523\#sthash.OBTOryTI.dpuf. Accessed June 16, 2015.

Lee, A. O. (1990). Airline Reservations Forecasting: Probabilistic and Statistical Models of the Booking Process. PhD dissertation, Massachusetts Institute of Technology.

Lieberman, W. H. (2004). Revenue management trends and opportunities. Journal of Revenue \& Pricing Management 3(1): 91-99.

Oliveira, V. M. (2003). Simulating revenue management in an airline market with demand segmentation and strategic interaction. Journal of Revenue \& Pricing Management 1(4): 301-318.

Ratliff, R. (1998). Ideas on overbooking. Presented at AGIFORS Reservations and Yield Management Study Group, Melbourne, Australia.

Rossi, P. E., G. M. Allenby and R. McCulloch. (2005). Bayesian Statistics and Marketing. John Wiley and Sons, Chichester, West Sussex, UK.

Talluri, K. T. and G. J. van Ryzin (2004a). The Theory and Practice of Revenue Management. Springer, New York.

Talluri, K. T. and G. J. van Ryzin. (2004b). Revenue management under a general discrete choice model of consumer behavior. Management Science 50(1): 15-33.

Train, K. E. (2009). Discrete Choice Methods with Simulation. Cambridge University Press, New York.

Veldwijk, J., M. S. Lambooij, E. W. de Bekker-Grob, H.A. Smit and G. A. de Wit. (2014). The effect of including an opt-out option in discrete choice experiments. PLOS ONE 9(11): e111805.doi:10.1371/journal.pone.0111805 
Westerhof, A. (1997). CO2 in the air. Presented at AGIFORS Reservations and Yield Management Study Group, Melbourne, Australia. 


\section{Appendix}

This appendix provides details on the Bayesian procedures used to estimate models with fixed parameters and models with random parameters.

\section{Estimation of the Model with Fixed Parameters}

Bayes estimation of the logit choice model exploits the following MCMC sampler, where all model parameters are updated using the Metropolis-Hastings algorithm with an independence chain (Rossi, et al. 2005):

1. Start with $\beta^{(g-1)}$ where $g$ is the iteration number.

2. Condition on $y$ and $X$ to find the maximum likelihood estimates $\hat{\beta}_{M L E}$.

3. Draw $\beta^{\text {cand }}$ from the multivariate $t$ proposal distribution $M S t\left(v, \hat{\beta}_{M L E}, S[\breve{T}+\right.$ $\left.\left.\mathcal{J}\left(\hat{\beta}_{M L E}\right)\right]^{-1} S\right) . S$ and $v$ are tuning parameters that can be adjusted to obtain an ideal acceptance rate. $\mathcal{J}$ is the Fisher information matrix of the logit model, and $\check{T}^{-1}$ is a prior precision.

4. Calculate the acceptance ratio

$$
\alpha=\min \left\{1, \frac{L\left(\beta^{\text {cond }} ; y \mid X\right) M S t\left(\beta^{\text {cond }}\right)}{L\left(\beta^{(g-1)} ; y \mid X\right) M S t\left(\beta^{(g-1)}\right)}\right\}
$$

5. Accept $\beta^{\text {cond }}=\beta^{(g)}$ with probability $\alpha$. Keep the old value with probability $(1-\alpha)$.

6. Update $g=g+1$, and go back to step 1 .

Note that in the model with fixed parameters all expanded binary choices are taken as independent, even if the sequence of binary choices represents a single ticket of the same person. The variability of the attribute levels over time is the only treatment of the dynamic structure of the problem. 


\section{Estimation of the Random Parameter Model}

Bayesian estimation of the random parameter model is described below, and follows the method outlined by Train (2009). As above, let the utility that a person $i$ obtains from alternative $j$ in time $t$ be

$$
U_{i j t}=\boldsymbol{x}_{i j t}^{\prime} \boldsymbol{\beta}_{i}+\varepsilon_{i j t}
$$

where $i=1, \ldots, n$ is independently and identically distributed extreme value, and $\beta_{i} \sim N(b, W)$. Assume that the prior on $b$ is normal with an unboundedly large variance, and the prior on $W$ is inverted Wishart $(I W)$ with $K$ degrees of freedom and scale matrix $I$, the $K$-dimensional identity matrix.

From equation (10) the probability of a person $i$ 's observed choices, conditional on $\beta$, is given by

$$
L\left(y_{i} \mid \beta\right)=\prod_{t} \frac{e^{x_{i y_{i t}}^{\prime} t^{\beta}}}{\sum_{j} e^{x_{i j t}^{\prime}}}
$$

The unconditional probability is thus given by the integral of $L\left(y_{i} \mid \beta\right)$ over all possible $\beta$,

$$
L\left(y_{i} \mid b, W\right)=\int L\left(y_{i} \mid \beta\right) \varphi(\beta \mid b, W) d \beta,
$$

where $\varphi(\beta \mid b, W)$ is the normal density with mean $b$ and variance $W$.

By definition, the posterior distribution of $b$ and $W$ is given as:

$$
K(b, W \mid Y) \alpha \prod_{i} L\left(y_{i} \mid b, W\right) k(b, W)
$$

where $k(b, W)$ is the prior on $b$ and $W$.

Although drawing directly from this posterior distribution is computationally slow when using the Metropolis-Hastings algorithm, it becomes fast and simple if each $\beta_{i}$ is seen as a parameter together with $b$ and $W$ and Gibbs sampling is used. Then the posterior from which draws are obtained becomes 


$$
K\left(b, W, \beta_{i} \forall i \mid Y\right) \alpha \prod_{i} L\left(y_{i} \mid \beta_{i}\right) \varphi\left(\beta_{i} \mid b, W\right) k(b, W)
$$

If we assume priors as stated before, then the Gibbs sampling proceeds as follows:

1. Start with $W^{(g-1)}, \beta_{i}^{(g-1)} \forall i$, where $g$ is the iteration number.

2. Draw $b^{(g)}$ from $N\left(\bar{\beta}^{(g-1)}, \frac{W^{(g-1)}}{n}\right)$, where $\bar{\beta}^{(g-1)}$ is the mean $\beta_{i}^{(g-1)} \forall i$.

3. Draw $W^{(g)}$ from $I W\left(K+n, \frac{K I+n S^{(g-1)}}{K+n}\right)$, where $S^{(g-1)}=\sum_{n} \frac{\left(\beta_{i}^{(g-1)}-b^{(g)}\right)\left(\beta_{i}^{(g-1)}-b^{(g)}\right)^{\prime}}{n}$.

4. Draw $\beta_{i}^{(g)} \forall i$, using one iteration of the Metropolis-Hastings algorithm described below.

5. Update $g=g+1$, and go back to Step 1 .

The Metropolis-Hastings algorithm with which $\beta_{i}^{(g)}$ is drawn proceeds as follows:

1. Start with $\beta_{i}^{(g-1)}$, where $g$ is the iteration number.

2. Draw $R$ independent values from a standard normal density, and stack these draws into vector $\eta^{(g)}$.

3. Let trail value $\tilde{\beta}_{i}^{(g)}=\beta_{i}^{(g-1)}+\rho L \eta^{(g)}$, where $\rho$ is a scaler pre-specified by the researcher, and $L$ is the Choleski factor of $W$.

4. Calculate the acceptance ratio

$$
\alpha=\min \left\{1, \frac{L\left(y_{i} \mid \widetilde{\beta}_{i}^{(g)}\right) \varphi\left(\widetilde{\beta}_{i}^{(g)} \mid b^{(g)}, W^{(g)}\right)}{L\left(y_{i} \widetilde{\beta}_{i}^{(g-1)}\right) \varphi\left(\widetilde{\beta}_{i}^{(g-1)} \mid b^{(g)}, W^{(g)}\right)}\right\}
$$

5. Accept $\beta_{i}^{(g)}=\tilde{\beta}_{i}^{(g)}$ with probability $\alpha$. Keep $\beta_{i}^{(g)}=\beta_{i}^{(g-1)}$ with probability $1-\alpha$. 


\section{List of Tables and Figures}

Table 1: Data format used for Bayesian estimation

Table 2: Variable definitions and descriptive statistics

Table 3: Comparison of classical and Bayesian estimates and odds ratios

Table 4: Validation that variables are randomly Normally distributed

Table 5: Decrease in cancellation rates due to termination of CVA

Figure 1: Individual means for probability of cancellation

Figure 2: Individual means and confidence intervals for probability of cancellation

Figure 3: Means and confidence intervals for probability of cancellation by day 
Table 1: Data format used for Bayesian Estimationestimation

\begin{tabular}{|c|c|c|c|c|c|c|c|c|}
\hline Customer & Choice & DFDI.1 & DFD1.2 & DFD0_3.1 & DFD0_3.2 & ... & A22.1 & A22.2 \\
\hline 52463 & 2 & 0 & 0 & 1 & 0 & & 1 & 0 \\
\hline 52463 & 2 & 1 & 0 & 1 & 0 & & 1 & 0 \\
\hline 52463 & 2 & 2 & 0 & 1 & 0 & & 1 & 0 \\
\hline 52463 & 2 & 3 & 0 & 1 & 0 & & 1 & 0 \\
\hline 52463 & 2 & 4 & 0 & 0 & 0 & & 1 & 0 \\
\hline 52463 & 2 & 5 & 0 & 0 & 0 & & 1 & 0 \\
\hline 52463 & 1 & 6 & 0 & 0 & 0 & & 1 & 0 \\
\hline
\end{tabular}


Table 2: Variable definitions and descriptive statistics

\begin{tabular}{|c|c|}
\hline $\begin{array}{l}\text { Variables (Notation } \\
\text { Used in Table 3) }\end{array}$ & Definition and Descriptive Statistics \\
\hline $\begin{array}{l}\text { Days from departure } \\
\text { (DFD) }\end{array}$ & $\begin{array}{l}\text { Number of days before the departure of the first leg of the outbound } \\
\text { itinerary that the ticket is booked. Ranges from } 0-238 \text { days; majority } \\
(65 \%) \text { is from } 1-21 \text { days. }\end{array}$ \\
\hline $\begin{array}{l}\text { Days from issue } \\
\text { (DFI) }\end{array}$ & $\begin{array}{l}\text { Number of days after ticket is issued that an event (defined as "cancel" } \\
\text { or "no cancel") occurs. Ranges from } 0-220 \text { days; majority of events } \\
\text { occur between 1-21 days from issue. }\end{array}$ \\
\hline $\begin{array}{l}\text { Departure day of week } \\
\text { (SUN, ..., SAT) }\end{array}$ & $\begin{array}{l}\text { Day of week on which the first flight of the outbound itinerary occurs. } \\
\text { Sun } 18 \% \text {; Mon } 22 \% \text {; Tues } 18 \% \text {; Wed } 18 \% \text {; Thurs } 14 \% \text {; Fri } 5 \% \text {; Sat } \\
5 \% \text {. }\end{array}$ \\
\hline $\begin{array}{l}\text { Departure month } \\
(\mathrm{JAN}, \ldots, \mathrm{DEC})\end{array}$ & $\begin{array}{l}\text { Month in which the first flight of the outbound itinerary occurs. Jan } \\
7 \% \text {; Feb 9\%; Mar 13\%; Apr 10\%; May 9\%; Jun 9\%; Jul 5\%; Aug 7\%; } \\
\text { Sept } 8 \% \text {; Oct 9\%; Nov 9\%; Dec 5\%. }\end{array}$ \\
\hline $\begin{array}{l}\text { Carrier } \\
(\mathrm{DL} \text { or FL) }\end{array}$ & $\begin{array}{l}\text { Marketing carrier associated with the ticket. Delta (DL) 97\%; AirTran } \\
\text { (FL) } 3 \% \text {. }\end{array}$ \\
\hline $\begin{array}{l}\text { ATL origin } \\
\text { (ATLORG) }\end{array}$ & $\begin{array}{l}\text { Indicator variable equal to } 1 \text { if first leg of the outbound itinerary } \\
\text { originated in Atlanta, } 0 \text { otherwise. } 81 \% \text { of tickets originate in Atlanta. } \\
\text { Reference=Atlanta is destination on ticket (e.g., would occur for } \\
\text { visitors to Georgia Tech). }\end{array}$ \\
\hline $\begin{array}{l}\text { State discount } \\
\text { (STATE) }\end{array}$ & $\begin{array}{l}\text { Indicator variable equal to } 1 \text { if the ticket involves a state discount (that } \\
\text { includes the ability to exchange ticket without a fee), } 0 \text { otherwise. } 40 \% \\
\text { receive State discount. }\end{array}$ \\
\hline $\begin{array}{l}\text { Length of stay } \\
(\text { LOS } 1, \text { LOS } 2, \ldots \\
\text { LOS7P ) }\end{array}$ & $\begin{array}{l}\text { Number of nights the passenger spends away from home, e.g., if the } \\
\text { first leg of the outbound trip starts on Monday and the first leg of the } \\
\text { inbound trip starts on Wednesday, the length of is } 2 . \text { Set to } 0 \text { for one- } \\
\text { way itineraries. Same day } 9 \% \text {; one } 25 \% \text {; two } 26 \% \text {; three } 19 \% \text {; four } \\
11 \% \text {; five } 5 \% \text {; six } 2 \% \text {; seven or more } 3 \% \text {. }\end{array}$ \\
\hline $\begin{array}{l}\text { Departure hour } \\
(\mathrm{D} 8, \mathrm{D} 9, \ldots \mathrm{D} 21)\end{array}$ & $\begin{array}{l}\text { Departure hour of the first leg of the outbound itinerary (in local time } \\
\text { of the origin airport). 83\% depart between 7:00 AM-5:59 PM; } \\
\text { distribution is fairly even across these departure hours. }\end{array}$ \\
\hline $\begin{array}{l}\text { Arrival hour } \\
(\mathrm{A} 8, \mathrm{~A} 9, \ldots, \mathrm{A} 22)\end{array}$ & $\begin{array}{l}\text { Arrival hour of the last leg of the outbound itinerary (in local time of } \\
\text { the destination airport). } 91 \% \text { arrive between 9:00 AM - 9:59 PM; most } \\
\text { popular arrival times are between 9:00 AM - 12:59 PM and 4:00 PM - } \\
\text { 7:59 PM. }\end{array}$ \\
\hline
\end{tabular}


Table 3: Comparison of classical and Bayesian estimates and odds ratios (OR)

\begin{tabular}{|c|c|c|c|c|}
\hline Parameter & $\begin{array}{c}\text { Class. } \\
\text { Est }\end{array}$ & $\begin{array}{c}\text { Bays. } \\
\text { Est }\end{array}$ & $\begin{array}{c}\text { Class. } \\
\text { OR }\end{array}$ & $\begin{array}{c}\text { Bays. } \\
\text { OR }\end{array}$ \\
\hline CONST*** & -5.68 & -5.74 & 0.00 & 0.00 \\
\hline DFI* & 0.01 & 0.01 & 1.01 & 1.01 \\
\hline DFI_0_3 & 0.18 & 0.17 & 1.20 & 1.19 \\
\hline DFI_4_7** & 0.47 & 0.47 & 1.60 & 1.60 \\
\hline DFI_8_14^ & 0.24 & 0.24 & 1.27 & 1.27 \\
\hline DFI_15_21* & 0.31 & 0.32 & 1.37 & 1.37 \\
\hline DFD $* * *$ & -0.04 & -0.04 & 0.96 & 0.96 \\
\hline ATLORG** & 0.32 & 0.32 & 1.37 & 1.38 \\
\hline STATE*** & 1.40 & 1.41 & 4.06 & 4.08 \\
\hline \multicolumn{5}{|c|}{ Departure Day of Week (Ref=Sat) } \\
\hline SUN & -0.10 & -0.08 & 0.91 & 0.92 \\
\hline MON & 0.09 & 0.11 & 1.10 & 1.11 \\
\hline TUES & 0.08 & 0.10 & 1.09 & 1.10 \\
\hline WED & -0.06 & -0.04 & 0.94 & 0.96 \\
\hline THURS & 0.00 & 0.01 & 1.00 & 1.01 \\
\hline $\mathrm{FRI}^{\wedge}$ & -0.41 & -0.41 & 0.66 & 0.66 \\
\hline \multicolumn{5}{|c|}{ Departure Month $($ Ref $=$ Dec $)$} \\
\hline JAN & -0.06 & $\begin{array}{l}-0.08 \\
\end{array}$ & 0.94 & 0.93 \\
\hline FEB & 0.00 & 0.01 & 1.00 & 0.99 \\
\hline MAR & -0.22 & -0.22 & 0.80 & 0.80 \\
\hline APR & -0.23 & -0.23 & 0.79 & 0.79 \\
\hline MAY & -0.24 & -0.24 & 0.79 & 0.79 \\
\hline JUN & -0.07 & -0.08 & 0.93 & 0.93 \\
\hline JUL & -0.28 & -0.28 & 0.76 & 0.76 \\
\hline AUG & 0.11 & 0.11 & 1.11 & 1.11 \\
\hline SEPT* & -0.45 & -0.45 & 0.64 & 0.64 \\
\hline OCT & 0.04 & 0.04 & 1.04 & 1.04 \\
\hline $\mathrm{NOV}^{\wedge}$ & -0.30 & -0.30 & 0.74 & 0.74 \\
\hline \multicolumn{5}{|c|}{ Marketing Carrier $($ Ref $=D L)$} \\
\hline $\mathrm{FL}^{\wedge}$ & -0.61 & $\begin{array}{l}-0.65 \\
\end{array}$ & 0.54 & 0.52 \\
\hline \multicolumn{5}{|c|}{ Length of Stay $($ Ref $=0$ days $)$} \\
\hline LOS1 & 0.03 & 0.03 & 1.03 & 1.03 \\
\hline LOS2 & 0.04 & 0.05 & 1.04 & 1.05 \\
\hline LOS3 & -0.16 & -0.15 & 0.85 & 0.86 \\
\hline LOS4 & -0.13 & -0.12 & 0.88 & 0.89 \\
\hline LOS5 & 0.06 & 0.05 & 1.06 & 1.06 \\
\hline LOS6 & -0.19 & -0.19 & 0.82 & 0.83 \\
\hline LOS7P & -0.06 & -0.07 & 0.94 & 0.93 \\
\hline $\begin{array}{l}\text { Log- } \\
\text { likelihood }\end{array}$ & -5403.4 & & & \\
\hline
\end{tabular}

\begin{tabular}{|l|r|r|r|c|}
\hline Parameter & $\begin{array}{c}\text { Class. } \\
\text { Est }\end{array}$ & $\begin{array}{c}\text { Bays. } \\
\text { Est }\end{array}$ & $\begin{array}{c}\text { Class. } \\
\text { OR }\end{array}$ & $\begin{array}{c}\text { Bays. } \\
\text { OR }\end{array}$ \\
\hline \hline Departure Hour $($ Ref $=10: 00 ~ P M-7: 59$ & AM $)$ \\
\hline \hline D8^ & -0.36 & -0.37 & 0.70 & 0.69 \\
\hline D9 & -0.01 & 0.03 & 0.99 & 0.97 \\
\hline D10 & 0.01 & 0.01 & 1.01 & 1.01 \\
\hline D11 & -0.40 & -0.41 & 0.67 & 0.67 \\
\hline D12 & -0.37 & -0.39 & 0.69 & 0.68 \\
\hline D13 & -0.31 & -0.31 & 0.74 & 0.73 \\
\hline D14 & -0.35 & -0.37 & 0.70 & 0.69 \\
\hline D15 & -0.13 & -0.13 & 0.88 & 0.88 \\
\hline D16 & -0.30 & -0.28 & 0.74 & 0.76 \\
\hline D17 & -0.36 & -0.35 & 0.70 & 0.70 \\
\hline D18 & -0.03 & 0.00 & 0.97 & 1.00 \\
\hline D19 & 0.45 & 0.48 & 1.57 & 1.62 \\
\hline D20 & 0.26 & 0.28 & 1.29 & 1.32 \\
\hline D21 & -0.42 & -0.45 & 0.66 & 0.64 \\
\hline \hline Arrival Hour $($ Ref $=11: 00 P M-7.59 A M)$ \\
\hline \hline A8 & -0.42 & -0.42 & 0.66 & 0.66 \\
\hline A9 & -0.19 & -0.19 & 0.82 & 0.83 \\
\hline A10^ & -0.52 & -0.51 & 0.60 & 0.60 \\
\hline A11 & -0.44 & -0.43 & 0.64 & 0.65 \\
\hline A12 & -0.49 & -0.49 & 0.61 & 0.61 \\
\hline A13 & -0.30 & -0.31 & 0.74 & 0.73 \\
\hline A14 & -0.44 & -0.45 & 0.64 & 0.63 \\
\hline A15 & -0.53 & -0.54 & 0.59 & 0.58 \\
\hline A16 & -0.40 & -0.41 & 0.67 & 0.66 \\
\hline A17 & -0.53 & -0.54 & 0.59 & 0.58 \\
\hline A18 & -0.18 & -0.19 & 0.84 & 0.83 \\
\hline A19 & -0.53 & -0.54 & 0.59 & 0.58 \\
\hline A20^ & -0.73 & -0.77 & 0.48 & 0.46 \\
\hline A21** & -1.21 & -1.24 & 0.30 & 0.29 \\
\hline A22^ & -0.72 & -0.77 & 0.49 & 0.46 \\
\hline
\end{tabular}

Significance Codes: $* * *=99.9 \% ; * *=99 \% ; *=95 \% ; \wedge=90 \%$.

Note: DFI_x_y= binary variable $=1$ if DFI is between $\mathrm{x}$ and y days; 0 o.w. 
Table 4: Random parameter estimates

\begin{tabular}{|c|c|c|}
\hline Variable & Mean & Std. Dev. \\
\hline \multicolumn{3}{|c|}{ RP1: Following Graham et al. (2010) } \\
\hline DFI_0_3*** & 0.68 & 0.13 \\
\hline DFI_4_7*** & 0.75 & 0.12 \\
\hline $\mathrm{DFD}^{* * *}$ & 0.10 & 0.0053 \\
\hline $\mathrm{FL}^{* * *}$ & 0.86 & 0.24 \\
\hline STATE*** & 0.90 & 0.087 \\
\hline Model Log-Likelihood & \multicolumn{2}{|c|}{-4076.63} \\
\hline \multicolumn{3}{|c|}{ RP2: Variables from binary logit model that are statistically significant at $95 \%$ level } \\
\hline DFI*** & 0.093 & 0.0041 \\
\hline DFI_4_7*** & 0.61 & 0.11 \\
\hline DFI_15_21*** & 0.61 & 0.14 \\
\hline $\mathrm{DFD} * * *$ & 0.098 & 0.0051 \\
\hline SEPT $* * *$ & 1.61 & 0.21 \\
\hline ATLORG $^{* * *}$ & 0.70 & 0.077 \\
\hline STATE*** & 0.61 & 0.10 \\
\hline $\mathrm{A} 21 * * *$ & 0.64 & 0.14 \\
\hline Model Log-Likelihood & \multicolumn{2}{|c|}{-3758.622} \\
\hline \multicolumn{3}{|c|}{ RP3: Variables from binary logit model that are statistically significant at $90 \%$ level } \\
\hline DFI*** & 0.096 & 0.0048 \\
\hline DFI_4_7*** & 0.44 & 0.053 \\
\hline DFI_8_14*** & 0.48 & 0.096 \\
\hline DFI_15_21*** & 0.72 & 0.12 \\
\hline $\mathrm{DFD} * * *$ & 0.10 & 0.0048 \\
\hline FRI*** & 0.88 & 0.21 \\
\hline SEPT*** & 1.26 & 0.17 \\
\hline NOV*** & 1.30 & 0.10 \\
\hline $\mathrm{FL} * * *$ & 1.25 & 0.32 \\
\hline $\mathrm{ATLORG}^{* * *}$ & 0.86 & 0.095 \\
\hline STATE*** & 0.74 & 0.083 \\
\hline $\mathrm{D} 8 * * *$ & 0.81 & 0.072 \\
\hline $\mathrm{A} 10 * * *$ & 1.67 & 0.13 \\
\hline $\mathrm{A} 20 * * *$ & 1.37 & 0.21 \\
\hline $\mathrm{A} 21 * * *$ & 0.61 & 0.12 \\
\hline $\mathrm{A} 22 * * *$ & 2.01 & 0.59 \\
\hline Model Log-Likelihood & \multicolumn{2}{|c|}{-3531.916} \\
\hline
\end{tabular}

Frequentist Significance Codes: $* * *=99.9 \% ; * *=99 \%$ 
Table 5: Decrease in cancellation rates due to termination of CVA

\begin{tabular}{|l|l|l||l|l|}
\cline { 2 - 5 } \multicolumn{1}{c|}{} & \multicolumn{2}{c||}{ With state rate (Prior to 1/1/2013) } & \multicolumn{2}{c|}{ Without state rate (Post 1/1/2013) } \\
\cline { 2 - 5 } \multicolumn{1}{c|}{} & Number & Percentage & Number & Percentage \\
\hline Tickets used & 8697 & $92.4 \%$ & 3398 & $95.7 \%$ \\
\hline Tickets cancelled & 711 & $7.6 \%$ & 152 & $4.3 \%$ \\
\hline Total & 9408 & $100 \%$ & 3550 & $100 \%$ \\
\hline
\end{tabular}




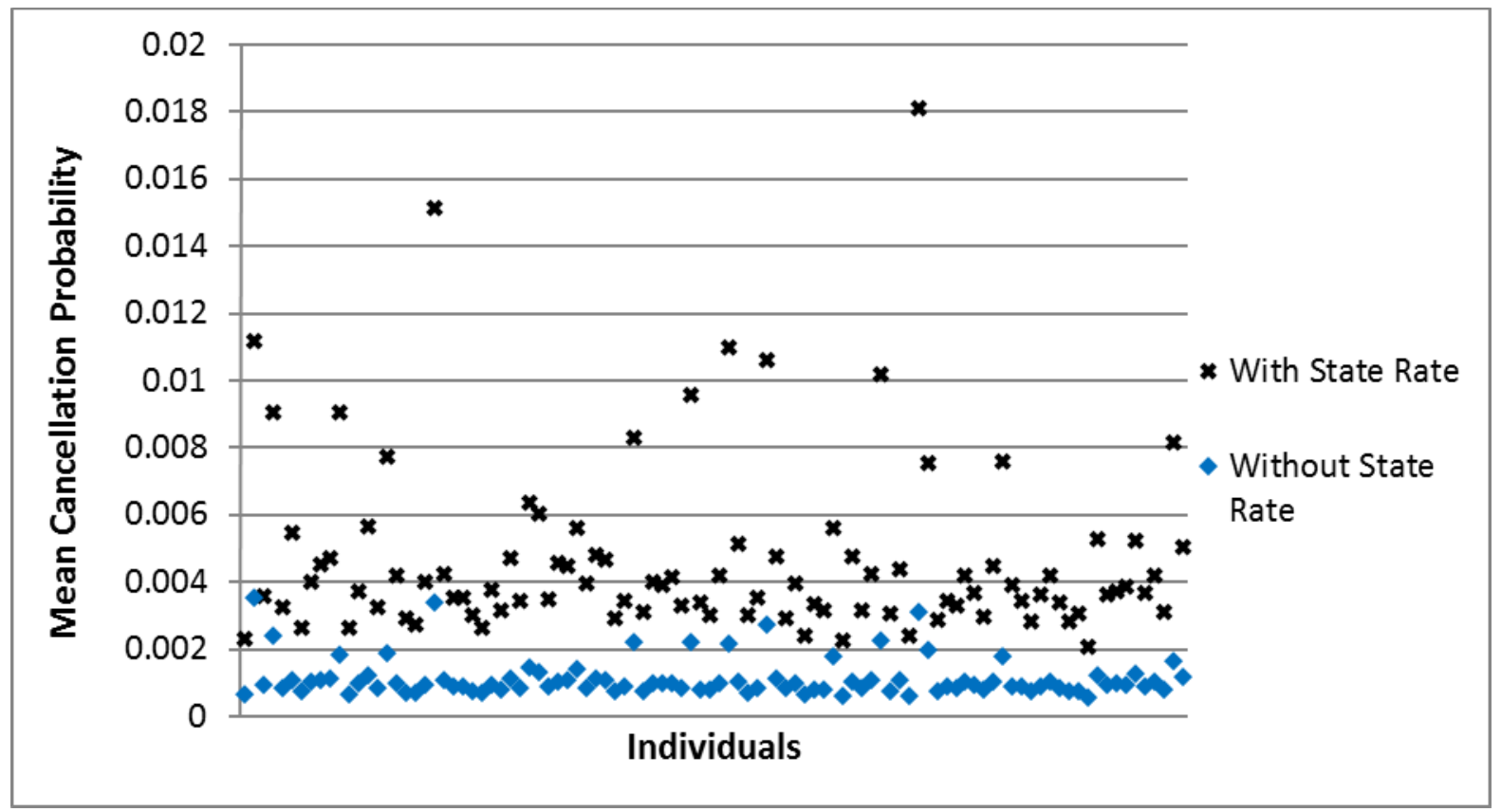

Figure 1: Individual posterior means for probability of cancellation 


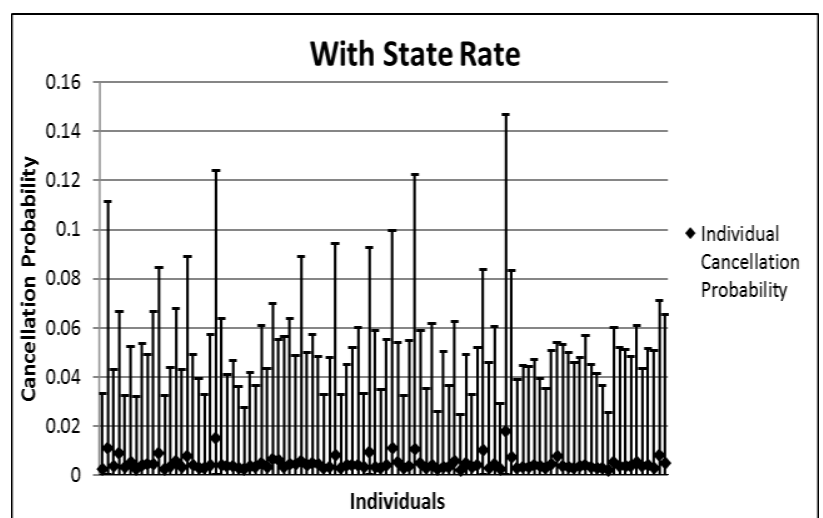

(a)

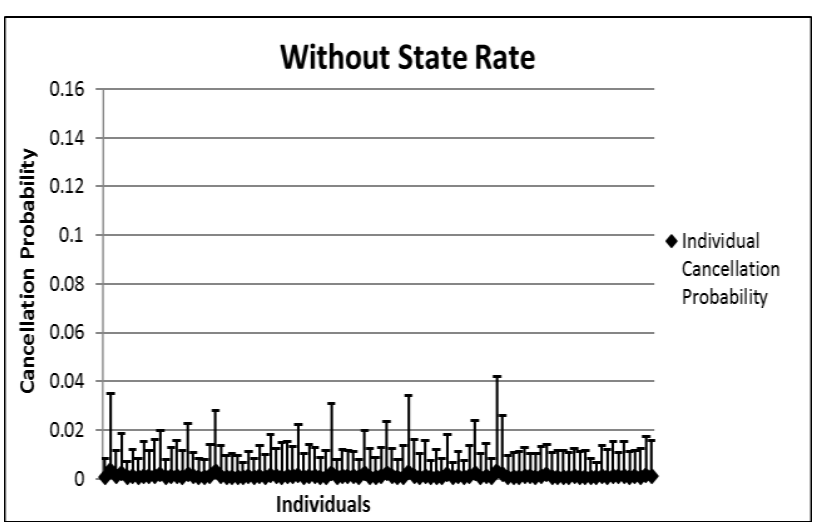

(b)

Figure 2: Individual posterior means and credible intervals for probability of cancellation 


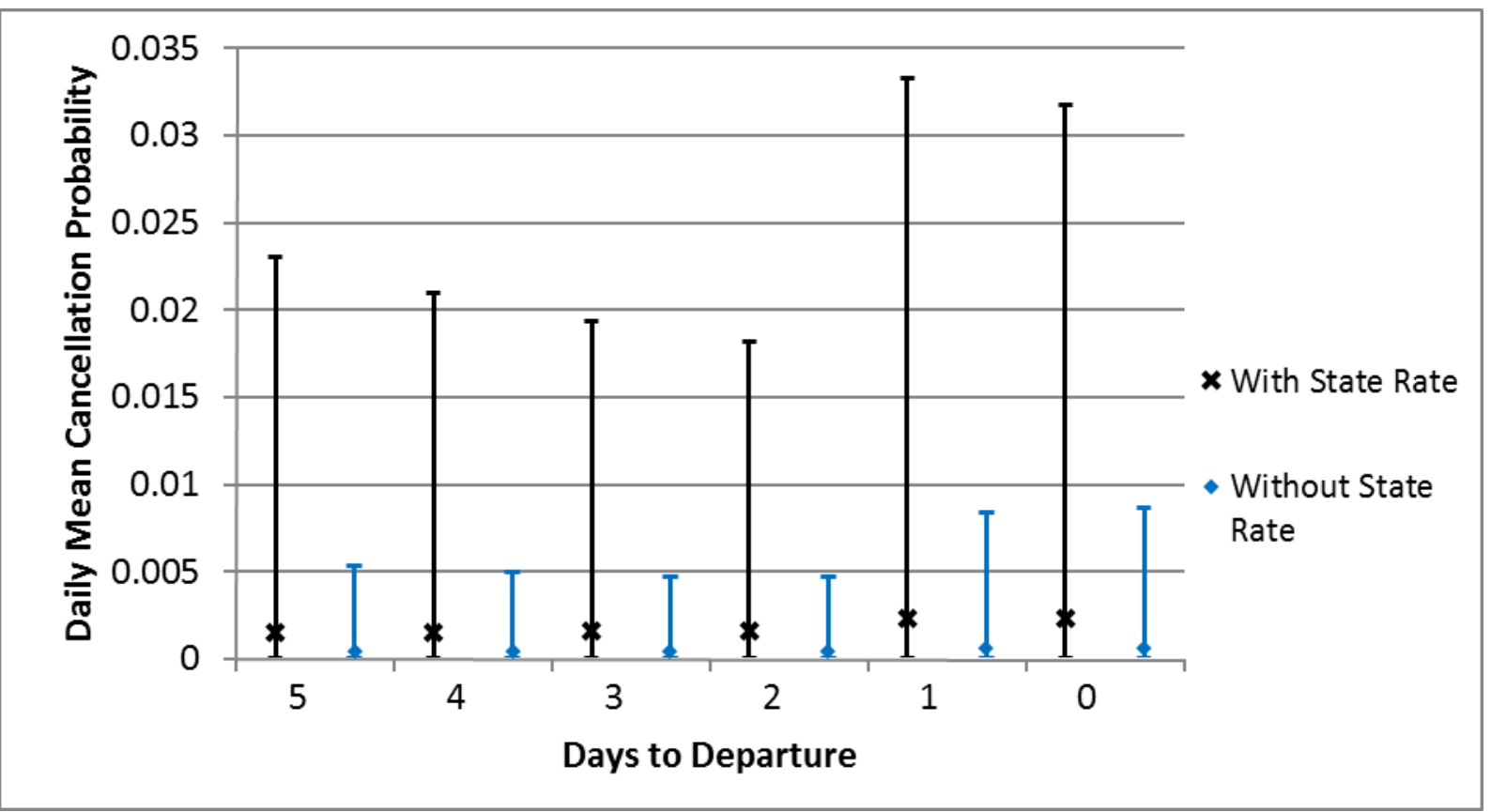

Figure 3: Posterior means and credible intervals for probability of cancellation by day 